\title{
Staphylococcus aureus isolado de swab nasal em um hospital militar
}

\author{
Staphylococcus aureus nasal swab of isolated in a military hospital \\ Maria Eduarda de Azevedo Goulart ${ }^{1}$, Cecília Santana Bala Pereira ${ }^{1}$, Lívia Machry², Saulo Roni \\ Moraes ${ }^{1,2}$.
}

Resumo

Como citar esse artigo. Goulart MEA, Pereira CSBP, Machry L, Moraes SR. Staphylococcus aureus nasal swab of isolated in a military hospital. Revista Saúde. 2015 Jan./Jun.; 06 (1): 05-28.

\begin{abstract}
Em razão do crescente surgimento de cepas de S. aureus resistentes a oxacilina (MRSA) surgiu o interesse em verificar a prevalência de MRSA isolados de swab nasal de pacientes internados no Hospital da ForçaAérea do Galeão-Rio de Janeiro(HFAG) e sua susceptibilidade a vancomicina. Trata-se de um estudo retrospectivo, de caráter quantitativo, descritivo e transversal baseado na análise dos resultados de exames de cultura qualitativa de swab nasal de pacientes internados no Hospital de Força Aérea do Galeão (HFAG) no Rio de Janeiro/RJ durante o período de janeiro a dezembro de 2011. Em 348 amostras analisadas, 298 (85,6\%) mostraram-se negativas para Staphylococcus aureus e 50 $(14,3 \%)$ mostraram-se positivas, desses, $29(42 \%)$ apresentaram resistência a oxacilina e $21(58 \%)$ não se mostraram resistentes. Referente à sensibilidadea vancomicina, $55 \%$ das cepas de S. aureus sensíveis a oxacilina(MSSA) apresentaram MIC $=2 \mu \mathrm{g} / \mathrm{ml}, 17,2 \%$ apresentaram MIC $=1 \mu \mathrm{g} / \mathrm{ml}, 13,7 \%$ apresentaram MIC $=0,5 \mu \mathrm{g} / \mathrm{mle} 13,7 \%$ apresentaram $\mathrm{MIC}=0,25 \mu \mathrm{g} / \mathrm{ml}$ para vancomicina. Em relação às cepas de $\mathrm{S}$. aureus resistentesa oxacilina, $52,3 \%$ apresentaramsensibilidadeavancomicinacomMIC $2 \mu \mathrm{g} / \mathrm{mle} 47,3 \%$ comMIC $1 \mu \mathrm{g} / \mathrm{ml}$. Pelo fatodorastreamento rotineiro do S. aureus ainda ocorrer apenas nos pacientes internado no HFAG, sugerimos que neste rastreamento seja também incluído os profissionais que atuam diretamente comos referidos pacientes eque seja discutidauma futura implantaçãode uma política de descontaminação dos pacientes e dos profissionais visando reduzir a disseminação de cepas de MRSA tanto na comunidade quanto dentro do hospital. Palavras-chave: Resistência a oxacilina. Staphylococcus aureus. Swab nasal.
\end{abstract}

\begin{abstract}
Due to the increasing emergence of strains of S. aureus resistant to oxacillin (MRSA) became interested in ascertaining the prevalence of MRSA isolates from nasal swabs of patients admitted to the Air Force Hospital Galeao - Rio de Janeiro (HFAG) and its susceptibility to vancomycin. This is a retrospective study of quantitative, descriptive and cross-sectional analysis based on the results of examinations of qualitative culture of nasal swabs from patients at the Hospital of the Air Force Galleon (HFAG) in Rio de Janeiro / RJ during the period January to December 2011. In 348 samples analyzed, 298 (85.6\%) were negative for Staphylococcus aureus and 50 (14.3\%) were positive, of which $29(42 \%)$ were resistant to oxacillin and $21(58 \%)$ sensitivity. To sensitivity to vancomycin, $55 \%$ of strains of S. aureus sensitive to oxacillin (MSSA) showed MIC $=2 \mathrm{mg} / \mathrm{ml}, 17.2 \%$ showed $\mathrm{MIC}=1 \mathrm{mg} / \mathrm{ml}, 13.7 \%$ showed MIC $=0.5 \mathrm{ug} / \mathrm{ml}$ and $13.7 \%$ showed MIC $=0,25 \mathrm{microg} / \mathrm{ml}$ for vancomycin. Regarding strains of $\mathrm{S}$. aureus resistant to oxacillin (MRSA), $52.3 \%$ were sensitive to vancomycin MIC $2 \mathrm{mg} / \mathrm{ml}$ and $47.3 \%$ with MIC $1 \mathrm{mg} / \mathrm{ml}$. Because the routine screening of S. aureus still occur only in patients hospitalized in HFAG, we suggest that this tracking is also included professionals who work directly with these patients and that is discussed a future implementation of a policy for decontamination of patients and professionals to reduce the spread of strains MRSA both in the community and within the hospital Keywords: Oxacillin resistance. Nasal swab. Staphylococcus aureus..
\end{abstract}

\section{Introdução}

Staphylococcus aureus é uma importante causa de infecções de tecidos moles e pele, bem como de infecções invasivas em humanos (Lowy, 1998). Desde que $S$. aureus resistente a meticilina (MRSA) foi relatado pela primeira vez, tem se tornado uma causa endêmica em hospitais e comunidades pelo mundo (Deresinski, 2005).

MRSA é o patógeno hospitalar resistente aos antibióticos mais prevalente em diversas partes do mundo, e existe um aumento do número de casos também na comunidade. Comparado com o Staphylococcus aureus sensível a meticilina (MSSA), infecções com MRSA são muito mais difíceis de tratar e tendem a desencadear pequenos surtos (Blot et al., 2002; Cosgrove et al., 2003).

S. aureus é um membro da microbiota anfibiôntica humana. As fossas nasais anteriores são o principal reservatório de colonização de $S$. aureus em humanos e muitas infecções por $S$. aureus ocorrem em pessoas que carream este microrganismo no nariz (Wertheim et al., 2005). A colonização nasal é uma importante etapa na patogênese das infecções por S. aureus e é um fator de risco para a aquisição de infecção nosocomial (Kluytmans, van Belkum, \& Verbrugh, 1997).

Tem sido demonstrado que $80 \%$ dos episódios de bacteremia nosocomial por $S$. aureus em carreadores desta bactéria foram atribuídos a uma fonte endógena (Von Eiff et al., 2001). Bacteremia nosocomial por $S$. aureus é três vezes mais freqüente em carreadores de $S$. aureus do que em não-carreadores (Wertheim et al., 2005). Vários estudos sobre o carreamento nasal de $S$. aureus tem sido realizado em várias regiões dos Estados Unidos e da Holanda (Bicshoff et al., 2002; Choi et al., 2006; Cole et al., 2001; Kilic et al., 2008; Kuehnert et al., 2006; Melles et al., 2008; Munckoff et al., 2009; Tenover et al., 2008). Levantamentos sobre os mecanismos

1. Universidade Severino Sombra, Centro de Ciências da Saúde, Farmácia, Vassouras-RJ, Brasil.

2. Farmacêutica do Setor de Análises Clínicas do Hospital da Força Aérea do Galeão, Rio de Janeiro-RJ, Brasil. 
de transmissão e a prevalência de carreadores nasais em especial nas populações sadias são benéficas para determinar os fatores de risco associados com infecções por S. aureus (Shopsin et al., 2000; Bicshoff et al., 2002; de Almeida Silva et al., 2003; Nules et al., 2005; Lowy et al., 2007; Farley et al., 2008; Sdougkos et al., 2008;).

O surgimento de uma cepa de MRSA associada à comunidade (CA-MRSA) altamente virulenta, cepas resistentes a vancomicina e cepas com resistência intermediária ou heteroresistentes de $S$. aureus, aumenta as preocupações da saúde pública (Hiramatisu et al., 1997; Wenzel \& Edmond, 1998; Smith et al, 1999; Gillet et al., 2002). A prevenção das infecções por $S$. aureus e a redução da disseminação de cepas virulentas e resistentes são, portanto, de grande importância.

Em razão do crescente surgimento de cepas de $S$. aureus resistentes a oxacilina (MRSA) surgiu o interesse em verificar a prevalência de MRSA isolados de swab nasal de pacientes internados no Hospital da Força Aérea do Galeão - Rio de Janeiro (HFAG) e sua susceptibilidade a vancomicina.

\section{Materiais e Métodos}

Trata-se um estudo retrospectivo, de caráter quantitativo, descritivo e transversal baseado na análise dos resultados de exames de cultura qualitativa de swab nasal de pacientes internados no Hospital de Força Aérea do Galeão (HFAG) no Rio de Janeiro/RJ durante o período de janeiro a dezembro de 2011. Os dados das culturas foram coletados em planilhas do setor de microbiologia do Laboratório de Análises Clínicas do referido hospital.

Swabs nasais foram obtidos de cada paciente pela rotação de um swab estéril, umidecido com salina estéril e introduzido a um centímetro de ambas as fossas nasais anteriores. Cada swab nasal foi semeado diretamente em placas de agar manita-sal e incubadas a $37^{\circ} \mathrm{C}$ por $24 \mathrm{~h}$ em aerobiose. Colônias suspeitas de $S$. aureus (colônias amarelas) foram identificadas através de processo semi-automatizado (Microsan $\left.{ }^{\circledR}\right)$. Além da identificação, o teste de susceptibilidade para diversos antibióticos, incluindo oxacilina e vancomicina foram determinados pelo mesmo método (CSLI, 2009).

\section{Resultados}

Das 348 amostras colhidas de swab nasal, 298 $(85,6 \%)$ mostraram-se negativas para Staphylococcus aureus e $50(14,3 \%)$ mostraram-se positivas (Gráfico $1)$.

Das 50 amostras positivas para Staphylococcus aureus, 29 (42\%) apresentaram resistência a oxacilina e $21(58 \%)$ sensibilidade a este antibiótico (Gráfico 2).
Gráfico 1. Freqüência de colonização por Staphylococcus aureus em amostras de swab nasal de pacientes internados no HFAG.

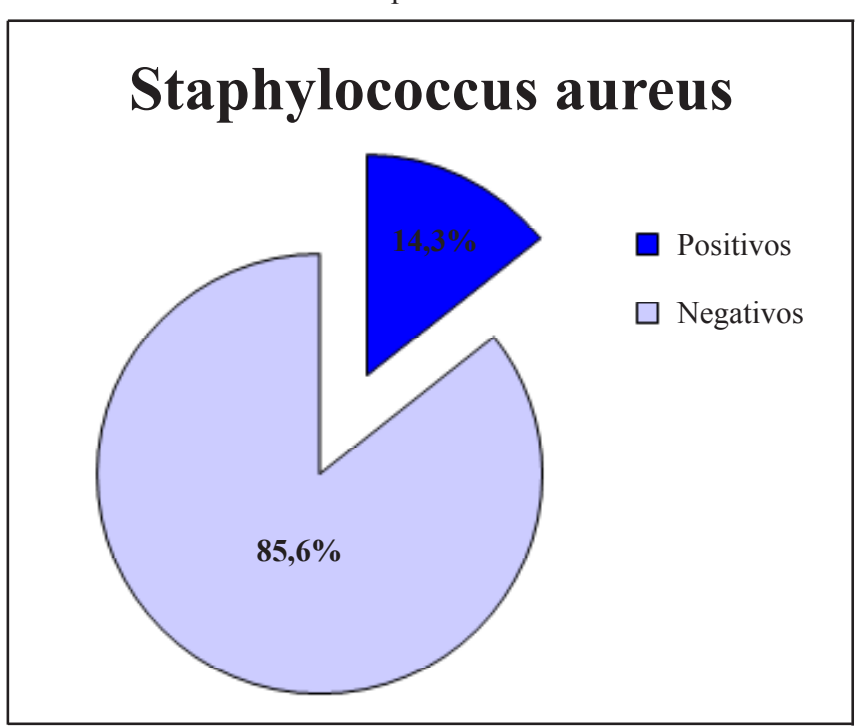

Gráfico 2. Percentual de MRSA E MSSA em exames positivos para Staphylococcus aureus obtidos de swab nasal de pacientes internados no HFAG.

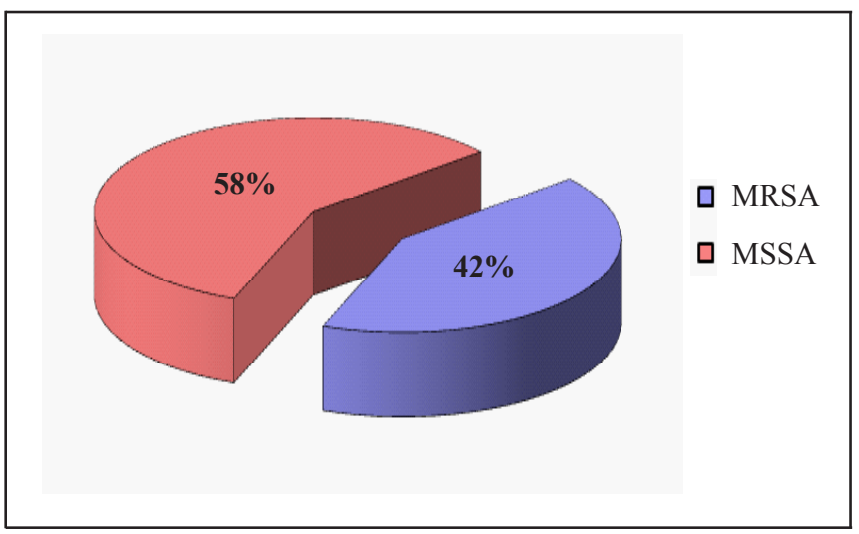

No presente estudo foi observado que $55 \%$ das cepas de $S$. aureus sensíveis a oxacilina (MSSA) apresentaram $\mathrm{MIC}=2 \mu \mathrm{g} / \mathrm{ml}, 17,2 \%$ apresentaram $\mathrm{MIC}=1 \mu \mathrm{g} / \mathrm{ml}, 13,7 \%$ apresentaram $\mathrm{MIC}=0,5 \mu \mathrm{g} /$ $\mathrm{ml}$ e $13,7 \%$ apresentaram $\mathrm{MIC}=0,25 \mu \mathrm{g} / \mathrm{ml}$ para vancomicina (Gráfico 3).

Gráfico 3. Perfil de sensibilidade do S. aureus sensível a oxacilina (MSSA) ao antibiótico vancomicina.

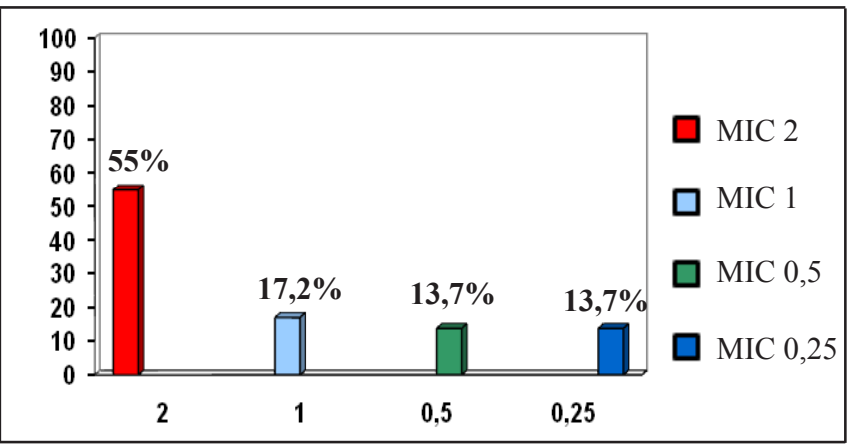


Em relação às cepas de $S$. aureus resistentes a oxacilina (MRSA), 52,3\% apresentaram sensibilidade a vancomicina com $\mathrm{MIC}=2 \mu \mathrm{g} / \mathrm{ml}$ e $47,3 \%$ com $\mathrm{MIC}=$ $1 \mu \mathrm{g} / \mathrm{ml}$ (Gráfico 4).

Gráfico 4. Perfil de sensibilidade de S. aureus resistente a oxacilina (MRSA) ao antibiótico vancomicina.

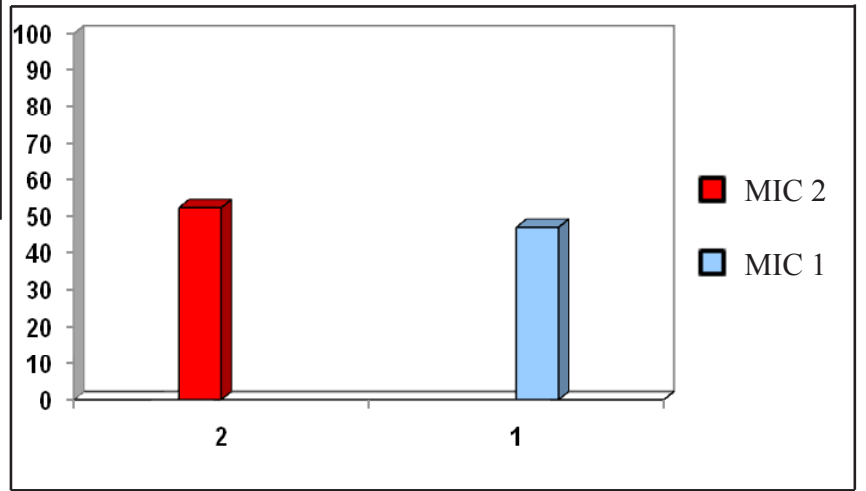

\section{Discussão}

Com a emergente disseminação mundial de $S$. aureus e evidências de transmissãocruzada entrehumanos, levantamentos sobre este patógeno tem se tornado crucial (Van Belkum \& Verbrugh, 1997; Kluytmans, Lowy, 1998; Ellis et al., 2004; Wertheim et al., 2005). Após avaliação da epidemiologia de colonização de $S$. aureus e dos fatores de risco, podem ser útil o desenvolvimento de estratégias efetivas de prevenção objetivando o controle da disseminação de MSSA e MRSA em pessoas saudáveis, especialmente dentro do ambiente hospitalar.

Em geral, S. aureus está presente no nariz de 20 a $30 \%$ das pessoas saudáveis (Kluytmans, van Belkum \& H. Verbrugh, 1997; Choi et al., 2005; Wertheim et al., 2005) e em maior freqüência na população que vive em aglomeração (Kenner et al., 2003; Bischoff et al., 2004; Ellis et al., 2004;). Sua taxa de carreamento nasal varia dependendo da população estudada. Nosso estudo indicou que ocorreu uma taxa de $14,3 \%$, similar ao ocorrido com 19,3\% dos pacientes internados na UTI do hospital público de Rio Branco - AC em 2009 (Garcez, 2011).

Dessas cepas de $S$. aureus, $42 \%$ apresentaram resistência a oxacilina (MRSA), semelhante ao ocorrido em hospitais de Portugal, onde apresentaram uma prevalência de 47 a 49\% (Valadas, 2010). Em um estudo realizado com pacientes de um hospital em Rio Branco AC em 2009 foi constatado que 41,7\% eram colonizados por MRSA (Garcez, 2011), o mesmo ocorreu com 31,1\% dos pacientes internados num hospital especializado em oncologia da cidade de São Paulo em 2011(Cataneo et al., 2011). Por outro lado, colonização por MRSA tem significantes variações na distribuição geográfica. As taxas de carreamento nasal de MRSA são maiores nos paises desenvolvidos.
O carreamento de MRSA é um pré-requisito para muitas infecções e exercem uma função importante na disseminação deste microrganismo dos ambientes de saúde e também na comunidade (Casewell \& Will, 1985; Casewell \& Will, 1986; Boyce, 1989; Darouiche et al., 1991). Na Holanda, devido ao programa de controle de infecção chamado "pesquisar e destruir" e uma política de controle severo sobre a antibiótico terapia, o número de pacientes colonizados com MRSA é ainda muito limitado (Wertheim et al., 2001; European Antimicrobial Resistance Surveillance System, 2007; Vos et al., 2009;). A etapa "Destruir" desta política é importante, pois visa eliminar dois dos três reservatórios conhecidos, a saber o paciente carreador e o profissional de saúde carreador, onde o terceiro reservatório seria o ambiente. Mas mesmo em baixa prevalência em paises como a Holanda, o surgimento de CA-MRSA tem causado uma mudança na epidemiologia e no aumento do número de casos por MRSA (European Antimicrobial Resistance Surveillance System, 2007).

As cepas $S$. aureus resistentes a vancomicina (VRSA) ainda são raras, mas o aumento de isolados com heterorresistência (hVISA) e resistência intermediária (VISA) tem sido reportado, inclusive no Brasil. Um estudo que avaliou as MICs de cepas MRSA diante da vancomicina, de 2001 a 2005, demonstrou que estas vêem aumentando, o que é motivo de preocupação. É relevante considerar que todas as VISA/hVISA provêm de pacientes previamente infectados com linhagens MRSA (Manfredini, Picoli, Becker, 2011).

No presente estudo, em relação a MSSA, foi observado que $55 \%$ de cepas apresentaram MIC $=2 \mu \mathrm{g} / \mathrm{ml}$. Em relação às cepas de MRSA, 52,3\% apresentaram $\mathrm{MIC}=$ $2 \mu \mathrm{g} / \mathrm{ml}$. Quando a MIC de vancomicina aumenta de $0,5 \mu \mathrm{g} /$ $\mathrm{ml}$ para $2 \mu \mathrm{g} / \mathrm{ml}$, eleva também o grau de falha terapêutica, mesmo que as cepas sigam categorizadas como sensíveis. Um trabalho que correlacionou MICs desse fármaco com eficácia clínica mostrou que nos isolados com MIC $\leq 1 \mu \mathrm{g} /$ $\mathrm{ml}$ o êxito do tratamento chega a $48 \%$, enquanto naquelas com valores $\geq 1 \mu \mathrm{g} / \mathrm{ml}$ o sucesso terapêutico não excede 17\% (Manfredini, Picoli, Becker, 2011).

O incremento da falha terapêutica em infecções causadas por isolados considerados sensíveis (MIC $\leq 2$ $\mu \mathrm{g} / \mathrm{ml}$ ) ocorre, principalmente, devido a cepas hVISA, ou seja, uma subpopulação com sensibilidade diminuída à vancomicina. Dessa forma, um resultado sensível pode não ser suficiente para o médico determinar o tratamento adequado (Cataneo et al., 2011; Manfredini, Picoli, Becker, 2011).

É importante mencionar que cepas de $S$. aureus podem apresentar sensibilidade a oxacilina e resistência a vancomicina. Em nosso hospital, uma cepa de $S$. aureus proveniente de uma amostra de aspirado endotraqueal apresentou o perfil de sensibilidade como o acima mencionado (Dados não publicados). Contudo, este resultado precisa de confirmação visto a raridade desta situação. 


\section{Referências}

BISCHOFF, W. E., WALLIS, M. L., TUCKER, K. B., REBOUSSIN, B. A., SHERERTZ, R. J. 2004. Staphylococcus aureus nasal carriage in a student community: prevalence, clonal relationships, and risk factors. Infect. Control Hosp. Epidemiol. 25:485-491.

BLOT, S. I., VANDEWOUDE ,K. H., HOSTE E. A., COLARDYN, F. A. 2002. Outcome and attributable mortality in critically ill patients with bacteremia involving methicillin-susceptible and methicillin-resistant Staphylococcus aureus. Arch. Intern. Med. 162:2229-2235.

BOYCE, J. M. 1989. Methicillin-resistant Staphylococcus aureus. Detection, epidemiology, and control measures. Infect. Dis. Clin. North Am. 3:901913.

CASEWELL, M. W., HILL R. L. 1986. Elimination of nasal carriage of Staphylococcus aureus with mupirocin ("pseudomonic acid") - a controlledtrial. J. Antimicrob. Chemother. 17:365-372.

CASEWELL, M. W., HILL, R. L. 1985. In-vitro activity of mupirocin ("pseudomonic acid") against clinical isolates of Staphylococcus aureus. J. Antimicrob. Chemother. 15:523-531.

CATANEO, C., CANINI, S., R., M., S., CASTRO, P., T., O., HAYASHIDA, M., GIR, E. 2011. Avaliação da sensibilidade e da especificidade dos critérios para isolamento de pacientes admitidos em um hospital especializado em oncologia. Rev. Latino-Am. Enfermagem. 19(5):[08 telas].

CHOI, C. S., YIN, C. S., BAKAR, A. A., SAKEWI, Z., NAING, N. N., JAMAL, F., OTHMAN, N. 2006. Nasal carriage of Staphylococcus aureus among healthy adults. J. Microbiol. Immunol. Infect. 39:458-464.

CLSI. 2009. Performance standards for antimicrobial susceptibility testing 10th informational supplement, M100-S18. Clinical and Laboratory Standards Institute, Wayne, PA.

COLE, A. TAHK, M., S., OREN, A., YOSHIOKA, D., KIM, Y. H., PARK, A. GANZ, T. 2001. Determinants of Staphylococcus aureus nasal carriage. Clin. Diagn. Lab. Immunol. 8:1064-1069.

COSGROVE, S. E., SAKOUlAS, G., PERENCEVICH, E. N., SCHWABER, M. J., KARCHMER, A. W., CARMELI, Y. 2003. Comparison of mortality associated with methicillin-resistant and methicillin-susceptible Staphylococcus aureus bacteremia: a meta-analysis. Clin. Infect. Dis. 36:5359.

DAROUICHE, R, C. WRIGHT, R, HAMILL, M. KOZA, D., LEWIS, MARKOWSKI 1991. Eradication of colonization by methicillin-resistant Staphylococcus aureus by using oral minocycline-rifampin and topical mupirocin. J. Antimicrob. Chemother. 35:1612-1615.

DE ALMEIDA SILVA, H., STEFFEN SBDALLAH, V. O., CARNEURO, C. L., GONTIJO, P. P. 2003. Infection and colonization by Staphylococcus aureus in a high risk nursery of a Brazilian teaching hospital. Braz. J. Infect. Dis. $7: 381-386$

DERESINSKI, S. 2005. Methicillin-resistant Staphylococcus aureus: an evolutionary, epidemiologic, and therapeutic odyssey. Clin. Infect. Dis. 40:562-573.

ELliS, M. W., HOSPENTHAL, D. R., DOOLEY, D. P., GRAY, P. J. MURRAY, C. K. 2004. Natural history of community-acquired methicillinresistant StaphylStaphylococcus aureus colonization and infection in soldiers. Clin. Infect. Dis. 39:971-979.

European Antimicrobial Resistance Surveillance System. 2007. EARSS Annual report 2006. EARSS, Bilthoven, The Netherlands. Disponível em: http://www.earssrivm.nl. Acesso em: 20 de setembro de 2012.

FARLEY, J. E., ROSS, T., STAMPER, P., BAUCOM, S., LARSON, E., CARROLL, K. C. 2008. Prevalence, risk factors, and molecular epidemiology of methicillinresistant Staphylococcus aureus among newly arrested men in Baltimore, Maryland. Am. J. Infect. Control 36:644-650.

GARCEZ, P., T., L., 2011. Estudo da ocorrência e perfil de susceptibilidade aos antimicrobianos de Staphylococcus aureus isolados de pacientes e profissionais de saúde na unidade de terapia intensiva de hospital público de
Rio Branco-AC. Belém - PA.

GILLET, Y, ISSARTEL, B, VANHEMS, P., FOURNET, J. C., LINA, G., BES, M., VANDENESCH, F., PIEMONT, Y., BROUSSE, N., FLORET, D., ETIENNE, J. 2002. Association between Staphylococcus aureus strains carrying gene for Panton- Valentine leukocidin and highly lethal necrotising pneumonia in young immunocompetent patients. Lancet 359:753-759.

HIRAMATSU, K., ARITAKA, N., HANAKI, H., KAWASAKI, S., HOSODA, Y., HORI, S., FUKUCHI, Y., KOBAYASHI, I. 1997. Dissemination in Japanese hospitals of strains of Staphylococcus aureus heterogeneously resistant to vancomycin. Lancet 350:1670-1673.

KENNER, J., O'CONNOR, T., PIANTANIDA, N., FISHBAIN, J., EBERLY, B., VISCOUNT, H., UYEHARA, C., HOSPENTAHL, D. 2003. Rates of carriage of methicillin resistant and methicillin-susceptible Staphylococcus aureus in an outpatient population. Infect. Control Hosp. Epidemiol. 24:439_ 444

KILIC, A., MERT, G., SENSES, Z., BEDIR, O., AYDOGAN, H., BASUSTAOGLU, A. C., APPELBAUM, P., C. 2008. Molecular characterization of methicillin-resistant Staphylococcus aureus nasal isolates from Turkey. Antonie Van Leeuwenhoek 94:615-619.

KLUYTMANS, J., VAN BELKUM, A., VERBRUGH, H. 1997. Nasal carriage of Staphylococcus aureus: epidemiology, underlying mechanisms, and associated risks. Clin. Microbiol. Rev. 10:505-520.

KUEHNERT, M. J., KRUSZON-MORAN, D., HILL, H. A., McQUILLAN, G., McAlLISTER, S. K., FOSHEIM, G., McDOUGAL, L. K., CHAITRAM, J., JENSEN, B., FRIDKIN, S. K., HILLGORE, G., TENOVER, F. C. 2006. Prevalence of Staphylococcus aureus nasal colonization in the United States, 2001-2002. J. Infect. Dis. 193:172-179.

LOWY, F. D. 1998. Staphylococcus aureus infections. N. Engl. J. Med. 339:520-532.

LOWY, F. D., AIELLO, A. E., BHAT, M., JOHNSON-LAWREWNCE, V. D., LEE, M. H., BURRELL, E., WRIGHT, L. N., VASQUEZ, G., LARSON, E. L. 2007. Staphylococcus aureus colonization and infection in New York State prisons. J. Infect. Dis. 196:911-918.

MANFREDINI, C., PICOLI, S., U., BECKER, A., P., 2011. Comparação de métodos na determinação de sensibilidade à vancomicina em Staphylococcus aureus resistente à meticilina. J Bras Patol Med Lab. v. 47. n. 2. p. 141-145.

MELLES, D. C., TENOVER, F. C., KUEHNERT, M. J., WITSENBOER, H., PEETERS, J. K., VERBRUGH, H. A., VAN BELKUM, A. 2008. Overlapping population structures of nasal isolates of Staphylococcus aureus from healthy Dutch and American individuals. J. Clin. Microbiol. 46:235-241.

MUNCKHOF, W. J., NIMMO, G. R., SCHOONEVELDT, J. M., SCHLEBUSCH, S., STEPHENS, A. J., WILLIAMS, G., HUYGENS, F., GIFFARD, P. 2009. Nasal carriage of Staphylococcus aureus, including community-associated methicillin-resistant strains, in Queensland adults. Clin. Microbiol. Infect. 15:149-155.

NULENS, E., GOULD, I., MAcKENZIE, F., DEPLANO, A., COOKSON, B., ALP, E., BOUZA, E., VOSS, A. 2005. Staphylococcus aureus carriage among participants

at the 13th European Congress of Clinical Microbiology and Infectious Diseases. Eur. J. Clin. Microbiol. Infect. Dis. 24:145-148.

PEACOCK, S. J., JUSTICE, A., GRIFFITHS, D., SILVA, G. D., KANTZANOU, M. N., CROOK, D., SLEEMAN, K., DAY, N. P. 2003. Determinants of acquisition and carriage of Staphylococcus aureus in infancy. J. Clin. Microbiol. 41:5718-5725.

SDOUGKOS, G., CHINI, V., PAPANASTASIOU, D. A., CHRISTODOULOU, G., STAMATAKIS, E., VRIS, A., CHRISTODOULIDI, I., PROTOPAPADAKIS, G., SPILIOPOUlOU, I. 2008. Communityassociated Staphylococcus aureus infections and nasal carriage among children: molecular microbial data and clinical characteristics. Clin. Microbiol. Infect. 14:995-1001.

SHOPSIN, B., MATHEMA, B., MARTINEZ, J., HA, E., CAMPO, M. L., FIERMAN, A., KRASINSKI, K., KORNBLUM, J., ALCABES, P., WADDINGTON, M., RIEHMAN, M., KREISWIRTH, B. N. 2000. Prevalence of methicillin-resistant and methicillin-susceptible Staphylococcus aureus in 
the community. J. Infect. Dis. 182:359-362.

SMITH, T. L., PEARSON, M. L., WILCOX, K. R., CRUZ, C., LANCASTER, M. V., ROBINSON-DUNN, B., TENOVER, F. C., ZERVOS, M. J., BAND, J. D., WHITE, E., JARVIS, W. R. 1999. Emergence of vancomycin resistance in Staphylococcus aureus. Glycopeptide Intermediate Staphylococcus aureus Working Group. N. Engl. J. Med. 340:493-501.

TENOVER, F. C., ARBEIT, R. D., GOERING, R. V., MICKELSEN, P. A., MURRAY, B. E., PERSING, D. H., SWAMINATHAN, B. 1995. Interpreting chromosomal DNA restriction patterns produced by pulsed-field gel electrophoresis: criteria for bacterial strain typing. J. Clin. Microbiol. 33:2233-2239.

VALADAS, M., M., B. 2010. Prevalência de colonização por MRSA no Centro Hospitalar P. Varzim/V. Conde. Disponível em: http://ria.ua.pt/ bitstream/10773/7634/1/Disserta\%C3\%A7\%C3\%A30\%20final.pdf. Acesso em: 16 de agosto de 2012.

VON EIFF, C., K. BECKER, K. MARCHKA, H. STAMMER, PETERS, G. 2001. Nasal carriage as a source of Staphylococcus aureus bacteremia. Study Group. N. Engl. J. Med. 344:11-16.

VOS, M. C., BEHRENDT, M. D., MELLES, D. C., MOLLEMA, F. P. N., GROOT, W., PARLEVLIET, G., OTT, A., HORST-KREFT, D., BELKUM, A, V. VERBRUGH, A. 2009. 5 years of experience implementing a methicillin-resistant Staphylococcus aureus search and destroy policy at the largest university medical center in The Netherlands. Infect. Control Hosp. Epidemiol. 30:977-984.

WENZEL, R. P., EDMOND, M. B. 1998. Vancomycin-resistant Staphylococcus aureus: infection control considerations. Clin. Infect. Dis. 27:245-249; quiz 250-251.

WERTHEIM, H. F., VOS, M. C., BOELENS, H. A., VOSS, A., VANDENBROUCKE GRAULS, C. M., MEESTER, M. H., KLUYTMANS, J. A., VAN KEULEN, P. H., VERBRUGH, H. A. 2004. Low prevalence of methicillin-resistant Staphylococcus aureus (MRSA) at hospital admission in the Netherlands: the value of search and destroy and restrictive antibiotic use. J. Hosp. Infect. 56:321-325.

WERTHEIM, H. F., MELLES, D. C., VOS, M. C., VAN LEEUWEN, W., VAN BELKUM, A., VERBRUGH, H. A., NOUWEN, J. L. 2005. The role of nasal carriage in Staphylococcus aureus infections. Lancet Infect. Dis. 5:751-762. 